\title{
Vertical diatomic artificial molecule in the intermediate-coupling regime in a parallel and perpendicular magnetic field
}

\author{
F. Ancilotto, ${ }^{1}$ D. G. Austing, ${ }^{2,5, *}$ M. Barranco,${ }^{3, \dagger}$ R. Mayol,${ }^{3}$ K. Muraki, ${ }^{2}$ M. Pi, ${ }^{3}$ S. Sasaki, ${ }^{2}$ and S. Tarucha ${ }^{2,4}$ \\ ${ }^{1}$ Istituto Nazionale per la Fisica della Materia and Dipartimento di Fisica, Università di Padova, I-35131 Padova, Italy \\ ${ }^{2}$ NTT Basic Research Laboratories, 3-1 Morinosato Wakamiya, Atsugi, Kanagawa, 243-0198, Japan \\ ${ }^{3}$ Departament ECM, Facultat de Física, Universitat de Barcelona, E-08028 Barcelona, Spain \\ ${ }^{4}$ Departament of Physics and ERATO Mesoscopic Correlation Project, University of Tokyo, \\ 7-3-1 Hongo, Bunkyo-ku, Tokyo, 113-0033, Japan \\ ${ }^{5}$ Institute for Microstructural Sciences M23A, National Research Council, Montreal Road, Ottawa, Ontario K1A OR6, Canada
}

(Received 8 July 2002; published 15 May 2003)

\begin{abstract}
We present experimental results for the ground-state electrochemical potentials of a few electron semiconductor artificial molecule made by vertically coupling two quantum dots, in the intermediate-coupling regime, in perpendicular and parallel magnetic fields up to $B \sim 5 \mathrm{~T}$. We perform a quantitative analysis based on local-spin density functional theory. The agreement between theoretical and experimental results is good, and the phase transitions are well reproduced.
\end{abstract}

DOI: 10.1103/PhysRevB.67.205311

PACS number(s): 73.21.-b, 85.35.Be, 36.40.Ei, 71.15.Mb

\section{INTRODUCTION}

Semiconductor quantum dots (QD's) are widely regarded as artificial atoms with properties analogous to those of "natural" atoms. Furthermore, systems composed of two QD's, "artificial” quantum molecules (QM's), coupled either laterally or vertically, have recently been investigated experimentally ${ }^{1}$ and theoretically. ${ }^{2-6}$ Transistors incorporating QM's (Ref. 7) made by vertically coupling two well defined and highly symmetric QD's (Ref. 8) are ideally suited to study QM properties. We recently reported the "addition energy" spectra at zero magnetic field for such QM's as a function of interdot coupling strength. ${ }^{9}$

In this work we present experimental and theoretical ground state electrochemical potentials for a diatomic QM in the intermediate-coupling regime corresponding to an interdot distance $b=3.2 \mathrm{~nm}$ for magnetic fields $(B)$ up to about 5 $\mathrm{T}$. We assume here that the quantum mechanical coupling is sufficiently strong that the QM can be regarded as a symmetric "homonuclear" diatomic QM. ${ }^{9}$ We consider two different configurations, one corresponding to an applied magnetic field parallel $\left(B_{\|}\right)$to the drain current $I_{d}$ flowing through the constituent QD's, and the other corresponding to an applied magnetic field perpendicular $\left(B_{\perp}\right)$ to $I_{d}$ (see Fig. 1). The latter has received relatively little attention. ${ }^{5,10-13}$ We note that the QM physics we discuss in both magnetic field configurations is particularly relevant to the subject of solid-state quantum computing. ${ }^{10}$

The interpretation of the experimental results here is based on the application of local-spin density-functional theory (LSDFT) ${ }^{6,14,15}$ In the $B_{\|}$case it follows the development of the method thoroughly described in Ref. 6, which includes finite thickness effects of the dots, and uses a relaxation method to solve the partial differential equations arising from a high order discretization of the Kohn-Sham (KS) equations on a spatial mesh in cylindrical coordinates (axial symmetry is assumed). To describe the less-common $B_{\perp}$ case, a three-dimensional (3D) LSDFT code has been devel- oped to handle configurations without any spatial symmetry.

This work is organized as follows. In Sec. II we describe the experimental setup; in Sec. III we outline the method used to implement LSDFT in our QM system and in Sec. IV we give the experimental and theoretical results. The interpretation of these results, and a short summary is presented in Sec. V.

\section{EXPERIMENT}

The molecules we study are formed by coupling, quantum mechanically and electrostatically, two QD's which individually display clear atomiclike features. ${ }^{7,8}$ For the materials we typically use, the energy splitting between the bonding and antibonding sets of single particle (s.p.) molecular states $\Delta_{\text {SAS }}$ can be varied from about $4.5 \mathrm{meV}$ (strong coupling) to about $0.1 \mathrm{meV}$ (weak coupling) ${ }^{6,7}$ In this paper, $b$, the thickness of the central barrier separating the two dots is fixed at $3.2 \mathrm{~nm}\left(\Delta_{\mathrm{SAS}} \sim 3 \mathrm{meV}\right)$. Because this corresponds to intermediate coupling, we can reasonably neglect a small mismatch (of energy $<\Delta_{\mathrm{SAS}}$ ) between the two dots, i.e., the QM is assumed to be symmetric "homonuclear." Figure 1 shows (a) a schematic section of a submicron circular mesa, diameter $D$, containing two vertically coupled QD's and (b) a

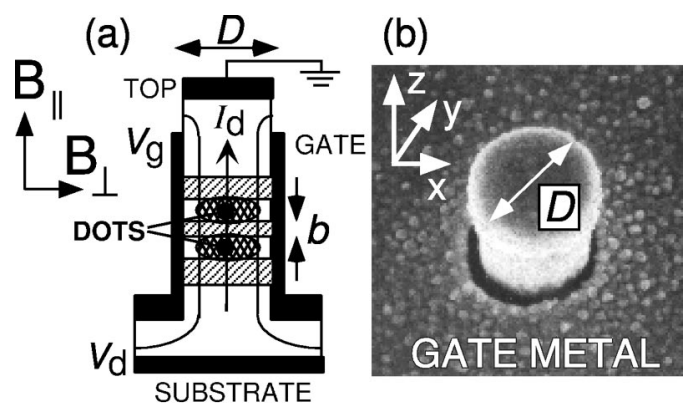

FIG. 1. (a) Schematic diagram of mesa containing two vertically coupled quantum dots and (b) scanning electron micrograph of a typical circular mesa. 


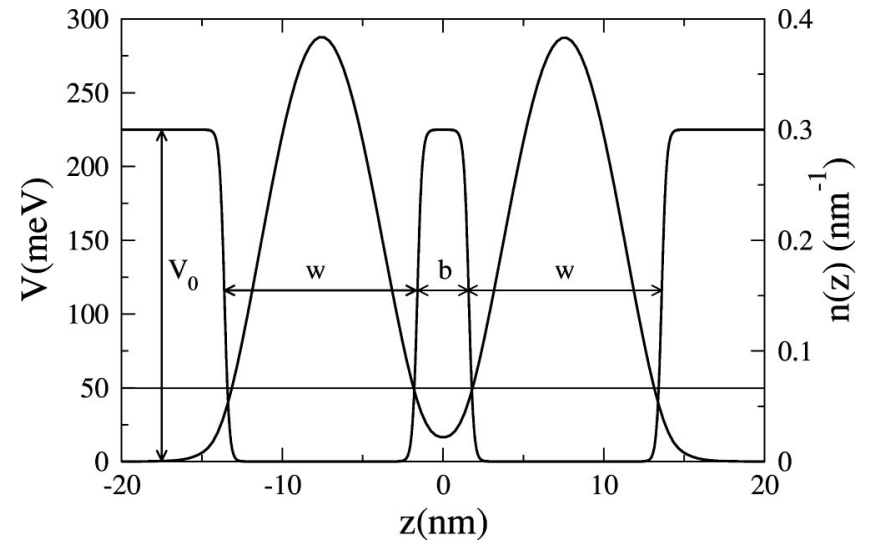

FIG. 2. Double quantum well potential used in the calculations. The electronic density $n(z)$ corresponding to the $N=6 \mathrm{QM}$ for $B$ $=0$ is shown. $n(z)$ has been obtained by integrating the electronic density $n(x, y, z)$ over the $x$ and $y$ coordinates. The energy of the occupied upper lying s.p. level is also represented by a horizontal solid line.

scanning electron micrograph of a typical mesa after gate metal deposition. The starting material, a special triple barrier resonant tunneling structure, and the processing recipe are described elsewhere. ${ }^{7,16,17}$ Drain current $I_{d}$ flows through the two QD's between the substrate contact and grounded top contact in response to voltage $V_{d}$ applied to the substrate and gate voltage $V_{g}$ on the single surrounding gate. The one structure we describe here ( $D \sim 0.5$ microns $)$ is cooled to about $300 \mathrm{mK}$ or less.

\section{THEORY}

To analyze the experiments we have modeled the QM by two identical QD's stacked in the $z$ direction (parallel to $I_{d}$ ). In this direction the QM is confined by two identical quantum wells of width $w$ and depth $V_{0}$ separated by distance $b$ $=3.2 \mathrm{~nm}$. We have taken $V_{0}=225 \mathrm{meV}$ and $w=12 \mathrm{~nm}$, which are also appropriate for the actual experimental device. To improve on the convergence of the $3 \mathrm{D}$ calculations, the somewhat ideal sharp double-well profile has been slightly rounded off, as shown in Fig. 2. Given that the energy profiles of real structures are never abruptly sharp, this rounding off is actually not unrealistic.

In the remaining two directions perpendicular to $z$ the QM is confined by a harmonic oscillator potential $V_{h}=m \omega^{2}\left(x^{2}\right.$ $\left.+y^{2}\right) / 2$ of fixed strength $\hbar \omega=4.42 \mathrm{meV}$. This lateral confinement energy has been determined for $N=6$ electrons using a law ${ }^{18}$ that quantitatively describes the phases of QM's in the strong, intermediate and weak coupling regimes as a function of $B_{\|}$for a number of electrons $N$ between 12 and 36. Lacking a better prescription at smaller $N, \hbar \omega$ has been kept fixed for all $N$ analyzed here $(N<7)$ instead of obscuring the results by further introducing an ad hoc $N$ dependency determined by a fitting procedure. ${ }^{19}$ In the following we will denote by $V_{\mathrm{cf}}(x, y, z)$ the total confining potential obtained by adding the double well profile to the harmonic oscillator potential $V_{h}$. We stress that $V_{\mathrm{cf}}(x, y, z)$ is axially symmetric around the $z$ axis.
As is well known, within LSDFT the ground state (g.s.) of the system is obtained by solving the Kohn-Sham (KS) equations. In the $B_{\|}$case the problem is greatly simplified by explicitly using the axial symmetry of the system. The additional terms in the KS equations due to the presence of an arbitrary magnetic field are given below. The inclusion of these terms crucially does not break the axial symmetry of the KS Hamiltonian in the $B_{\|}$case.

In the symmetric gauge the vector potential $\vec{A}(\vec{r})$ corresponding to a constant magnetic field $\vec{B}$ is written as $\vec{A}$ $=(\vec{B} \wedge \vec{r}) / 2$, and its contribution to the KS Hamiltonian is

$$
\mathcal{H}_{m}=\frac{e \hbar}{2 m c} \vec{B} \cdot \vec{L}+\frac{e^{2}}{2 m c^{2}} \vec{A}^{2}+g_{s}^{*} \mu_{B} \vec{B} \cdot \vec{S}
$$

where $g_{s}^{*}$ is the effective gyromagnetic factor, $\vec{L}$ and $\vec{S}$, respectively, are the orbital and spin angular momentum operators, and $\mu_{B}$ is the Bohr magneton. Writing $\vec{B}$ $=B\left(\sin \theta_{B}, 0, \cos \theta_{B}\right)$ and introducing the cyclotron frequency $\omega_{c}=e B / m c$, it can be easily checked that $\mathcal{H}_{m}$ $=\mathcal{H}_{m_{R}}+i \mathcal{H}_{m_{I}}$, with

$$
\begin{aligned}
\mathcal{H}_{m_{R}}= & \frac{1}{8} m \omega_{c}^{2}\left[x^{2} \cos ^{2} \theta_{B}+y^{2}+z^{2} \sin ^{2} \theta_{B}-2 x z \sin \theta_{B} \cos \theta_{B}\right] \\
& +\frac{1}{2} g_{s}^{*} \mu_{B} \eta_{\sigma} B
\end{aligned}
$$

$\mathcal{H}_{m_{I}}=-\frac{1}{2} \hbar \omega_{c}\left[\sin \theta_{B}\left(y \frac{\partial}{\partial z}-z \frac{\partial}{\partial y}\right)+\cos \theta_{B}\left(x \frac{\partial}{\partial y}-y \frac{\partial}{\partial x}\right)\right]$,

where $\eta_{\sigma}=+1(-1)$ for $\sigma=\uparrow(\downarrow)$ with respect to the direction of the applied magnetic field.

We have used effective atomic units $\hbar=e^{2} / \epsilon=m=1$, where $\epsilon$ is the dielectric constant and $m$ the electron effective mass. In units of the bare electron mass $m_{e}, m=m^{*} m_{e}$. In this system, the length unit is the effective Bohr radius $a_{0}^{*}$ $=a_{0} \epsilon / m^{*}$ with $a_{0}=\hbar^{2} / m_{e} e^{2}$, and the energy unit is the effective Hartree $H^{*}=H m^{*} / \epsilon^{2}$. For a QD in GaAs, we take the following values: $g_{s}^{*}=-0.44, \epsilon=12.4$, and $m^{*}$ $=0.067$. This yields $a_{0}^{*} \sim 97.94 \AA$ and $H^{*} \sim 11.86 \mathrm{meV}$. From now on we will write the equations in these units.

Equation (2) reduces to the $B_{\|}$case when $\theta_{B}=0$, and to the $B_{\perp}$ case when $\theta_{B}=\pi / 2$. In the former, since $[x(\partial / \partial y)$ $-y(\partial / \partial x)]$ is proportional to $L_{z}$, the problem remains axially symmetric. A detailed description of how the KS equations have been solved in this geometry can be found in Ref. 6.

In $3 \mathrm{D}$ the $\mathrm{KS}$ equations read

$$
\begin{gathered}
{\left[-\frac{1}{2}\left(\frac{\partial^{2}}{\partial x^{2}}+\frac{\partial^{2}}{\partial y^{2}}+\frac{\partial^{2}}{\partial z^{2}}\right)+V_{\mathrm{cf}}(x, y, z)+V^{H}+V^{\mathrm{xc}}\right.} \\
\left.+W^{\mathrm{xc}} \eta_{\sigma}+\mathcal{H}_{m}\right] \Psi_{\sigma}(x, y, z)=\epsilon_{\sigma} \Psi_{\sigma}(x, y, z) .
\end{gathered}
$$


The expression in the brackets is the KS Hamiltonian $\mathcal{H}_{\mathrm{KS}}$, and $V^{H}(x, y, z)$ is the direct Coulomb potential. $V^{\mathrm{xc}}$ $=\delta \mathcal{E}_{\mathrm{xc}}(n, m) /\left.\delta n\right|_{\text {g.s. }}$, and $W^{\mathrm{xc}}=\delta \mathcal{E}_{\mathrm{xc}}(n, m) /\left.\delta m\right|_{\text {g.s. }}$ are, respectively, the variation of the exchange-correlation energy density $\mathcal{E}_{\mathrm{xc}}(n, m)$ in terms of the electron g.s. density $n(x, y, z)$, and of the local spin magnetization $m(x, y, z)$ $\equiv n^{\uparrow}(x, y, z)-n^{\downarrow}(x, y, z)$. The exchange-correlation energy has been taken from Perdew and Zunger, ${ }^{14}$ and $\mathcal{E}_{\text {xc }}(n, m)$ has been constructed as indicated in Ref. 6 . It is worth noticing that if $B \neq 0$ then the s.p. wave functions $\Psi_{\sigma}(x, y, z)$ are complex, with their real and imaginary parts being coupled by $\mathcal{H}_{m}$.

The KS and Poisson equations are solved on a 3D mesh after discretizing them using seven-point formulas, and using a two-grid version of the one-way multigrid method described in Ref. 20. The Poisson equation is solved using a first order relaxation scheme. ${ }^{21}$ The required value of the Coulomb potential at the mesh boundary is obtained by a standard multipole expansion up to eighth order. The KS equations are solved using an imaginary time method, involving the third-order expansion of the forward solution of the imaginary time diffusion equation ${ }^{21}$

$$
\frac{\partial \Psi}{\partial \tau}=-\left(\mathcal{H}_{\mathrm{KS}}-\epsilon\right) \Psi
$$

i.e.,

$$
\begin{aligned}
\Psi(\tau+\delta \tau)-\Psi(\tau) \equiv & \Delta \Psi(\tau) \\
= & -\delta \tau\left(\mathcal{H}_{\mathrm{KS}}-\epsilon\right) \Psi(\tau)+\frac{\delta \tau^{2}}{2}\left(\mathcal{H}_{\mathrm{KS}}-\epsilon\right)^{2} \\
& \times \Psi(\tau)-\frac{\delta \tau^{3}}{6}\left(\mathcal{H}_{\mathrm{KS}}-\epsilon\right)^{3} \Psi(\tau),
\end{aligned}
$$

where $\epsilon=\left\langle\Psi(\tau)\left|\mathcal{H}_{\mathrm{KS}}\right| \Psi(\tau)\right\rangle$. To further accelerate the selfconsistent solution of both the KS and Poisson equations, we use the preconditioning smoothing operation described in Ref. 22. In the KS case, this means that $\Delta \Psi(\tau)$ has been smoothed as proposed in this reference. The performance of the code has been further improved by adding a "viscosity term," i.e., Eq. (5) has been changed into

$$
\Psi(\tau+\delta \tau)-\Psi(\tau)=\Delta \Psi(\tau)+\alpha_{V}[\Psi(\tau)-\Psi(\tau-\delta \tau)] .
$$

A viscosity term has also been included in the solution of the Poisson equation. We have used a $45 \times 45 \times 67$ mesh with spatial steps $\Delta x=\Delta y=5.67 \mathrm{~nm}$ and $\Delta z=0.89 \mathrm{~nm}$. The large asymmetry between the spatial meshes is motivated by the sharpness of the confining potential in the $z$ direction. The heuristic viscosity parameter $\alpha_{V}$ is fixed to a value of 0.8 , and the time step $\delta \tau$ to the value of $(\Delta z)^{2}$ in effective atomic units. The stability of our results against the increase of the number of mesh points and of the order of the formulas used to discretize the partial derivatives has been checked, and at $B=0$ we have used the results of the axially symmetric code to test the results obtained with the 3D code. This is a rather stringent test, since in the $3 \mathrm{D}$ case we have started the iteration procedure for solving the KS and Pois-

\begin{tabular}{|c|c|c|c|c|c|c|}
\hline \multirow[b]{2}{*}{$N$} & \multicolumn{2}{|c|}{ 2D-CSDFT } & \multicolumn{2}{|c|}{ 2D-LSDFT } & \multicolumn{2}{|c|}{ 3D-LSDFT } \\
\hline & $L(\mathrm{~T})$ & $R(\mathrm{~T})$ & $L(\mathrm{~T})$ & $R(\mathrm{~T})$ & $L(\mathrm{~T})$ & $R(\mathrm{~T})$ \\
\hline 20 & 5.6 & 6.3 & 5.4 & 6.5 & 5.4 & 6.4 \\
\hline 28 & 5.6 & 6.1 & 5.5 & 6.2 & 5.5 & 6.1 \\
\hline 36 & 5.6 & 5.9 & 5.5 & 6.0 & 5.6 & 6.0 \\
\hline
\end{tabular}

TABLE I. Comparison between two-dimensional CSDFT and LSDFT, and three-dimensional LSDFT results for one single QD. $L(R)$ denotes the left(right) border of the MDD phase in the $N-B_{\|}$ plane.

son equations from random number wave functions. As in Ref. 6, we have also used as a test the comparison between the total energy calculated from a straightforward integration of the energy density with the expression in terms of the s.p. energies derived from the KS equations. Finally, we have tested $a$ posteriori the validity of the multipole expansion of the Coulomb potential comparing the results with those obtained using fast Fourier transform techniques to evaluate it.

The accuracy of LSDFT for the $B$ values of interest has been assessed by comparing the results for a single QD with those obtained using the current spin-density functional theory (CSDFT), ${ }^{23-27}$ which in principle is better suited to high magnetic fields than LSDFT. Since CSDFT is a twodimensional (2D) theory, ${ }^{23}$ we have also compared our LSDFT results with those obtained using the 2D-LSDFT which is implicit in any implementation of CSDFT, in particular see that of Ref. 24. The low and high field borders of the maximum-density droplet (MDD) phase using strictly 2DLSDFT and CSDFT, as described in Ref. 24, have been obtained for QD's with $N=20,28$ and 36 electrons laterally confined by a harmonic oscillator potential of energy $\hbar \omega$ $=7.6 N^{-1 / 4}(\mathrm{meV})$. Such parametrization of the confining potential within LSDFT reproduces the experimental MDD. ${ }^{28}$ The results are shown in Table I, together with the values obtained by using the present 3D-LSDFT for the same lateral confining potential. From Table I we can see that the overall agreement between the three calculations is clearly good, and thus we can confidently use LSDFT in the present calculations.

\section{RESULTS AND DISCUSSION}

The experimental ground state electrochemical potentials for $N=3$ to 6 , as a function of $B$, are shown in Fig. 3 for (a) the parallel case and (b) the perpendicular case. What is actually shown is the $B$-field dependence of the third, fourth, fifth, and sixth current (Coulomb oscillation) peaks measured by sweeping $V_{g}$ in the linear conductance regime for a small $V_{d} \simeq 0.1 \mathrm{mV}$. It is clearly evident that the dependencies for parallel and perpendicular cases are very different-in particular the former is stronger than the latter. We now attempt to explain the general appearance in both cases and, in particular, the features marked by the different symbols, by using the computational methods described in the previous section. 

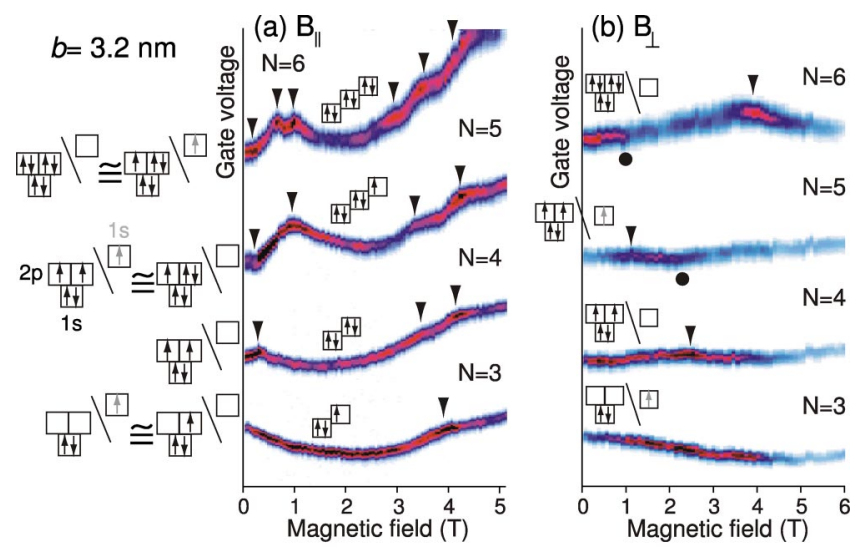

FIG. 3. Experimental $B$-field dependence of the third to sixth Coulomb oscillation peaks (g.s. electrochemical potentials for 3 $\leqslant N \leqslant 6$ ) in (a) $B_{\|}$case and (b) $B_{\perp}$ case.

\section{A. The $B_{\|}$case}

As in single QD's, at low $B$ fields, upward kinks (cusps) in the experimental $N$ electron g.s. QM electrochemical potentials as a function of $B$ are interpreted as changes in the $N$ electron g.s. configuration of the QM which arise from s.p. level crossings. ${ }^{8,28,29}$ We have plotted in Fig. 4 the calculated g.s. electrochemical potential $\mu(N)$, defined as

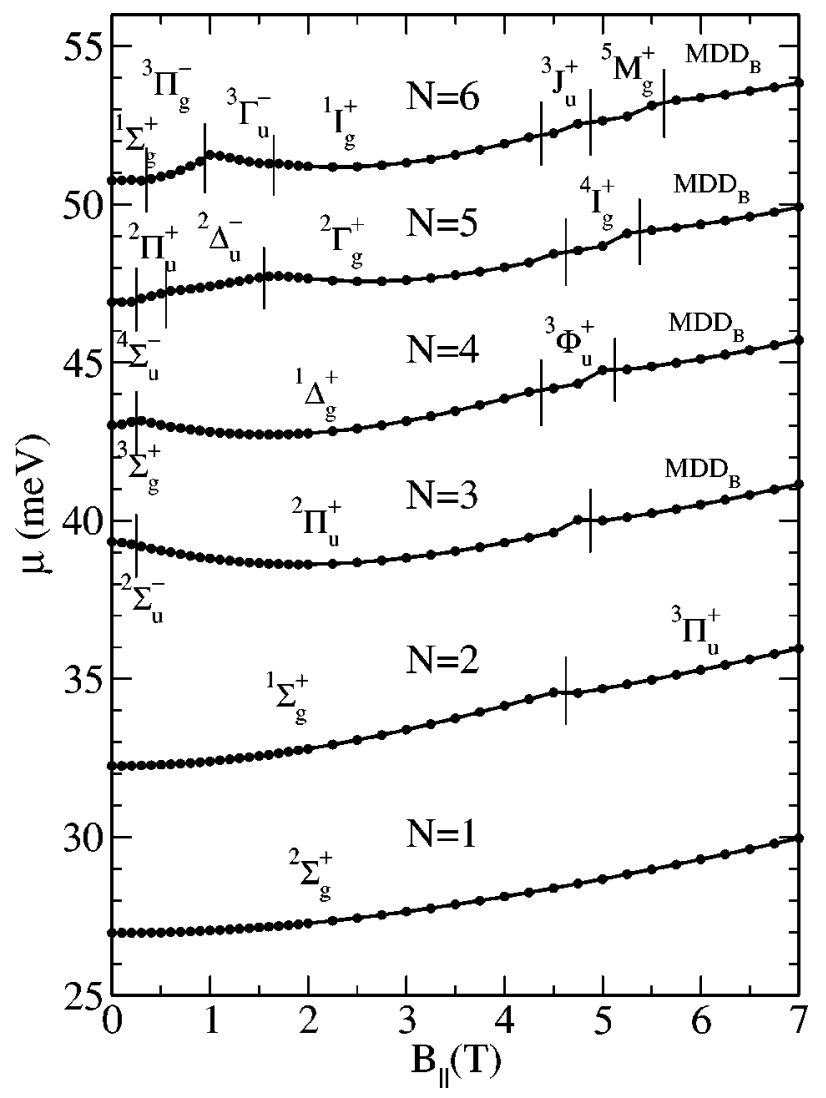

FIG. 4. Theoretical ground state electrochemical potentials in the $B_{\|}$case (dots) for $N \leqslant 6$. The lines have been drawn as a to guide the eye. The vertical ticks along the $\mu(N)$ lines indicate phase boundaries. The various states are identified by standard spectroscopic notation discussed in the text.

$$
\mu(N)=U(N)-U(N-1)
$$

where $U$ is the total energy of the $N$ electron QM g.s., as a function of $B_{\|}$up to $N=6$. To label the g.s. configurations we have used the usual notation of molecular physics for s.p. electronic orbitals. ${ }^{30}$ Upper case Greek letters are used for the total orbital angular momentum. We have also used the adapted version ${ }^{3}$ of the ordinary spectroscopic notation ${ }^{2 S+1} L_{g, u}^{ \pm}$with $S$ being the total $\left|S_{z}\right|$ and $L$ being the total $\left|L_{z}\right|$. The superscript $+(-)$ refers to even (odd) states under reflection with respect to the $z=0$ plane bisecting the QM. Even states are bonding (symmetric) states, and odd states are antibonding (antisymmetric) states. The subscript $g(u)$ refers to positive (negative) parity states. All these are good quantum numbers in the $B_{\|}$case and can be used to label the different g.s.'s ('phases"). Following Refs. 4,27 we have also calculated the "isospin" quantum number (the bond order in molecular physics $\left.{ }^{31}\right)$ defined as $I_{z}=\left(N_{B}-N_{A B}\right) / 2$, with $N_{B(A B)}$ being the number of electrons in bonding (antibonding) s.p. states. This is an exact quantum number for homonuclear QM's in a parallel magnetic field.

Given the complexity of real vertical QM structures and the challenge in modeling them, ${ }^{3,4,9,27}$ a comparison between Figs. 3(a) and 4 reveals a rather good agreement between theory and experiment. As a guide, and consistent with the calculated states and the observed $B_{\|}$dependence, we indicate in Fig. 3(a) in simple box style ${ }^{30}$ the dominant g.s. configurations at or near $B=0$, and others at higher field which are stable over a relatively wide range of $B_{\|}$. Up and down arrows indicate spin-up and spin-down electrons, and black (gray) arrows represent electrons in bonding (antibonding) s.p. states. ${ }^{29,30}$ For $N=3,5,6$, near $B=0$, because the g.s.'s are close to each other, i.e. stable over a fairly narrow range, we show two configurations which in practice are hard to resolve. Some of these involve the population of the lowest antibonding state with a single electron, so isospin is nonmaximal. Above $B=1 \mathrm{~T}$, however, all the antibonding states are depopulated so isospin is maximal $\left(I_{z}=N / 2\right)$, and filling of the QM resembles that of a single QD. The identifiable g.s. transitions in Fig. 3(a) are marked by black triangles. As expected, most appear as upward kinks. A couple, see first kinks for $N=5$ and 6 , appear as downward kinks because of the g.s. transitions which occur at almost the same $B_{\|}$in $N$ $=4$ and 5 , respectively.

Looking further at other details in Fig. 4, for $N=2$, the singlet-triplet transition occurs at about $4.6 \mathrm{~T}$ which is close to the experimental value ${ }^{17}$ of $\sim 4.2 \mathrm{~T}$ (not shown). We have found from the calculations a MDD configuration made of electrons filling just bonding s.p. states $\left(\mathrm{MDD}_{B}\right)$, which has a total angular momentum $L_{z}=N(N-1) / 2$, and extends from $\sim 4.9$ to $\sim 9.5 \mathrm{~T}$ for $N=3$, from $\sim 5.1$ to $\sim 9.0 \mathrm{~T}$ for $N=4$, from $\sim 5.4$ to $\sim 8.8 \mathrm{~T}$ for $N=5$, and from $\sim 5.6$ to $\sim 8.3 \mathrm{~T}$ for $N=6$. These results are at variance with those of Ref. 27, where an $\mathrm{MDD}_{B}$ g.s. was found for $N=3$, but not for larger values of $N$. The reason of this discrepancy may be attributed either to the strictly 2D model used in their calculation to represent the constituent QD's, or more likely to their particular implementation of CSDFT. ${ }^{32}$ 

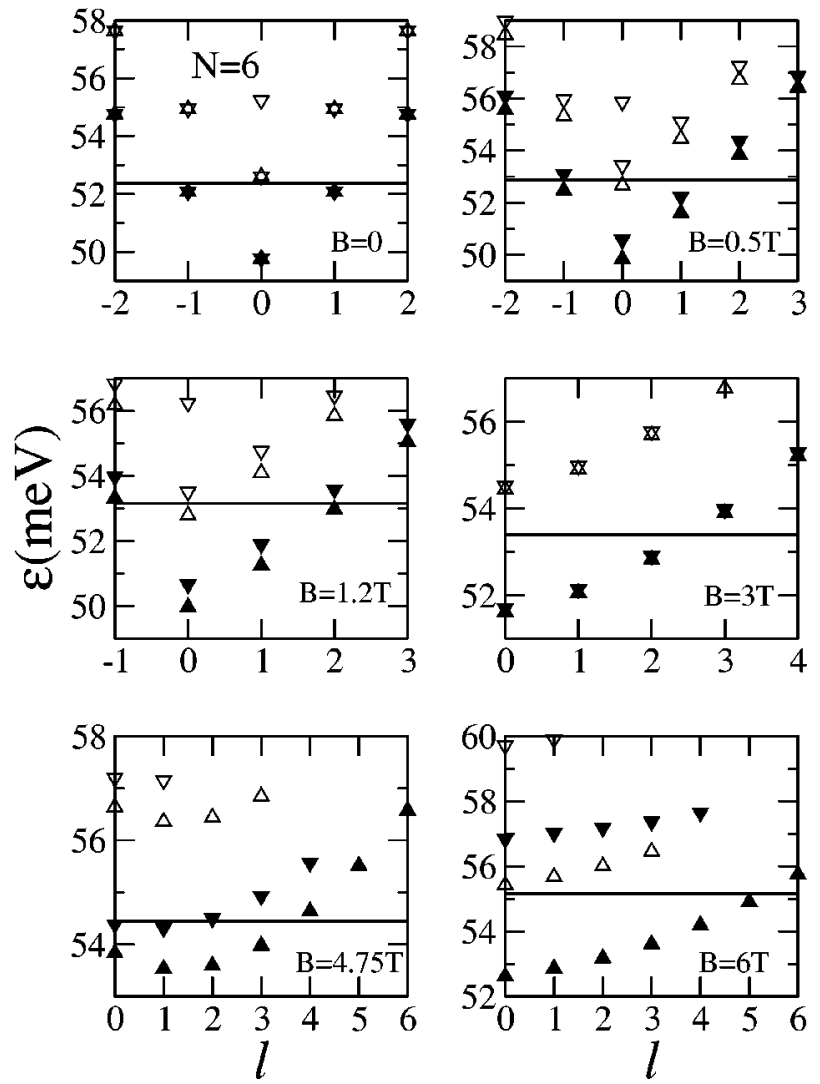

FIG. 5. Single particle energy levels as a function of $l$ for different values of $B_{\|}$at $N=6$. Upward(downward) triangles denote $\uparrow(\downarrow)$ spin states. Open(solid) triangles correspond to antibonding(bonding) states. The horizontal lines represent the Fermi level. The value of $B_{\|}$is indicated in each panel.

In Fig. 4 we can see that for the larger $N$ values studied here, the increase in angular momentum of the QM g.s. as it evolves from $B=0$ towards the $\mathrm{MDD}_{B}$ is accompanied by two isospin flips ${ }^{27}$ caused by electrons jumping from antibonding to bonding states and vice versa. Phase transitions from - to + g.s.'s involve $\Delta I_{z}=1$ flips, whereas those from + to - g.s.'s involve $\Delta I_{z}=-1$ flips, and both are clearly seen in Fig. 4 for $N=5$ and 6. Interestingly, they only happen for $B_{\|}<2 \mathrm{~T}$. We can see that after reaching the $\nu_{B}=2$ g.s. (i.e., a filling factor $2 \mathrm{QM}$ state made of just bonding s.p. states), which corresponds to the ${ }^{1} \Delta_{g}^{+}$phase for $N=4$, to the ${ }^{2} \Gamma_{g}^{+}$phase for $N=5$, and to the ${ }^{1} I_{g}^{+}$phase for $N=6$, only bonding s.p. states are occupied, and as a consequence the QM reaches the $\mathrm{MDD}_{B}$ state in a similar way to how a single QD reaches the MDD state, namely, by populating bonding s.p. states of higher and higher s.p. orbital angular momentum $l$ values. ${ }^{33}$ In general, these isospin flips can produce a complex pattern in the s.p. spectrum as a function of $B_{\|}$. As an example of this complexity, we present in Fig. 5 the s.p. levels for $N=6$ as a function of $l$ for different $B_{\|}$values. It can be seen in this figure that as $B$ increases, the QM undergoes isospin flips. First, the $l=0 \uparrow$ antibonding s.p. state becomes occupied, as shown in the panels corresponding to $B$ $=0.5$ and 1.2 T. After another isospin flip caused by the depopulation of the same s.p. state, the QM reaches the $\nu_{B}$

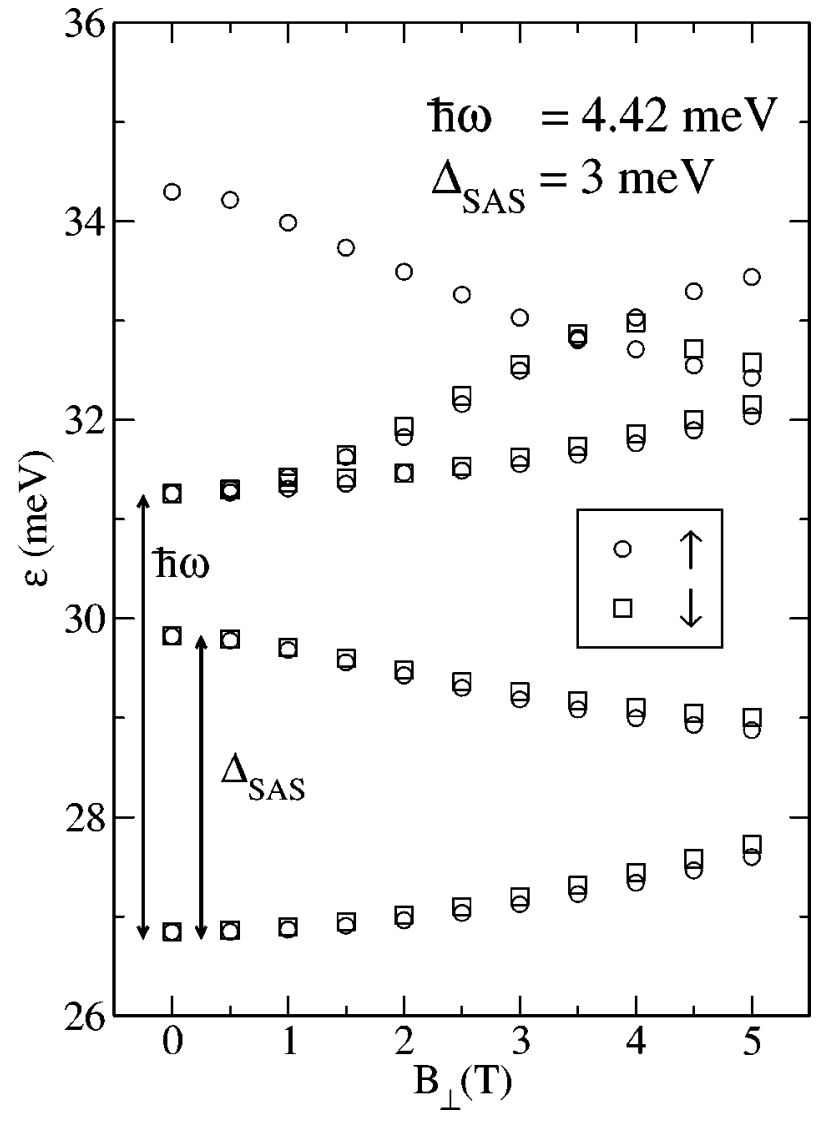

FIG. 6. Energies of the nine lower-lying noninteracting s.p. levels as a function of $B_{\perp} . \Delta_{\mathrm{SAS}}$ and $\hbar \omega$ are marked. For each symbol, the direction of $s_{\perp}$ is indicated in the box.

$=2$ phase corresponding to the ${ }^{1} I_{g}^{+}$configuration $(B=3 \mathrm{~T}$ panel). From this phase on, the spin polarization steadily increases until the $\mathrm{QM}$ reaches the $\mathrm{MDD}_{B}$ phase $(B=6 \mathrm{~T}$ panel).

\section{B. The $B_{\perp}$ case}

In the $B_{\perp}$ case, even if the experimental device is axially symmetric about the $z$ axis and the constituents QD's are identical, the magnetic Hamiltonian (1) breaks the axial symmetry and the reflection symmetry about the $z=0$ plane. As a consequence, the s.p. states no longer have a well defined orbital angular momentum nor parity, and the bonding or antibonding labels strictly do not make sense. Crucially, within LSDFT, the only good quantum number is the spin projection along the direction of the applied magnetic field, which we call $s_{\perp}$, and the g.s. electrochemical potentials as a function of $B_{\perp}$ are expected to be much smoother than in the $B_{\|}$case.

The situation of a $B_{\perp}$ field $^{5,10-13}$ unlike the $B_{\|}$case, lacks an analytical solution even for the case of noninteracting electrons. We show in Fig. 6 the calculated noninteracting s.p. spectrum as a function of $B_{\perp}$. At $B=0$ (and only in this case), the energy difference between the bonding and antibonding $l=0$ s.p. states is just $\Delta_{\mathrm{SAS}}$ (likewise for the $l=1$ states). Also, the energy difference between $l=1$ and 0 bonding (or antibonding) states is just $\hbar \omega$. Similar results have 


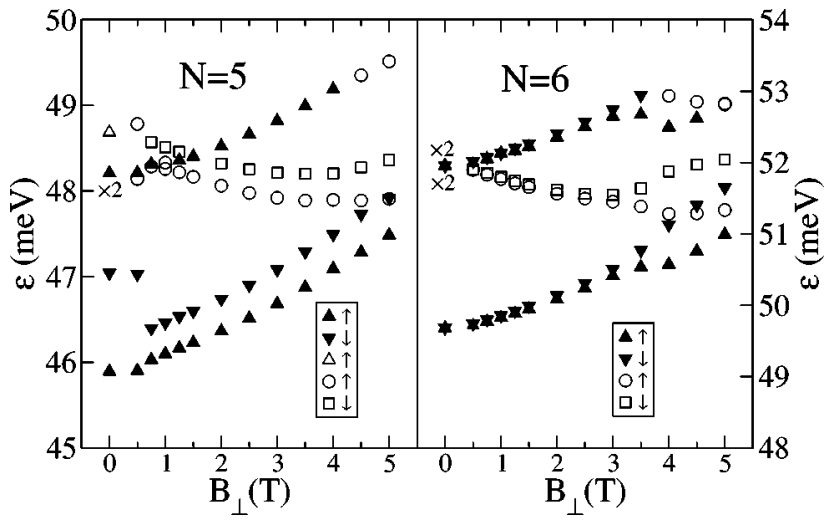

FIG. 7. Single particle energy levels as a function of $B_{\perp}$ for $N$ $=5$ (left panel) and 6 (right panel). For each symbol, the direction of $s_{\perp}$ is indicated in the box. States twofold degenerate are indicated by $\times 2$ symbol. Solid and open symbols are discussed in the text.

been reported elsewhere. ${ }^{5,13}$ The small splitting between spin up and down states that originate from a common s.p. state with well defined orbital angular momentum at $B=0$ is due to the Zeeman term.

As already noted, the even (bonding) or odd (antibonding) character of the s.p. levels defining a QM state is strictly lost when a magnetic field perpendicular to $I_{\mathrm{d}}$ is present. Intriguingly, however, the bonding/antibonding character present at $B=0$ is sometimes retained to a large degree by the s.p. states at finite $B_{\perp}$ values. We have indeed found that the expectation value of the $z \rightarrow-z$ reflection operator

$$
\left\langle\Pi_{z}\right\rangle=\int d \vec{r} \Psi^{*}(\vec{r}) \Pi_{z} \Psi(\vec{r})=\int d \vec{r} \Psi^{*}(x, y, z) \Psi(x, y,-z)
$$

is very close to \pm 1 , as it should be for bona fide bonding/ antibonding states, in many cases even for relatively large values of $B_{\perp}$.

As an example of this, we show in Fig. 7 the energies of the occupied s.p. states as a function of $B_{\perp}$ for $N=5$ and 6 . Solid triangles represent "quasibonding" states with $\left\langle\Pi_{z}\right\rangle$ $\geqslant 0.95$. Note that at $0 \mathrm{~T}$ for $N=5$ the s.p. bonding state at $\epsilon \sim 48.2 \mathrm{meV}$ is twofold degenerate, and likewise for two of the $N=6$ s.p. bonding states at $\epsilon \sim 52 \mathrm{meV}$. Open triangles represent "quasiantibonding" states with $\left\langle\Pi_{z}\right\rangle \leqslant-0.95$. Actually, there is only one such occupied antibonding s.p. state for $N=5$ at $B=0$, and none for $N=6$. All other open symbols (circles and squares) correspond to s.p. states with negative $\left\langle\Pi_{z}\right\rangle$ values larger than -0.95 , i.e., cannot really be regarded even as quasiantibonding states.

The figure also shows that states that evolve from $l=0$ s.p. states at $B=0$ retain a quasibonding character up to quite high values of $B_{\perp}$ (at least up to $5 \mathrm{~T}$ ), whereas other states, that at $B=0$ are $l=1$ s.p. states, do not. The quasibonding robustness of the lower-lying s.p. states may be due to the small effect that the applied magnetic field has on states that are $l=0 \mathrm{~s}$.p. states at $B=0$. The $B_{\perp}$ evolution of what at $0 \mathrm{~T}$ are the $2 p$ states is rather similar for $N=5$ and 6 with a change from solid to open symbols near $B_{\perp}=4 \mathrm{~T}$. Interest-

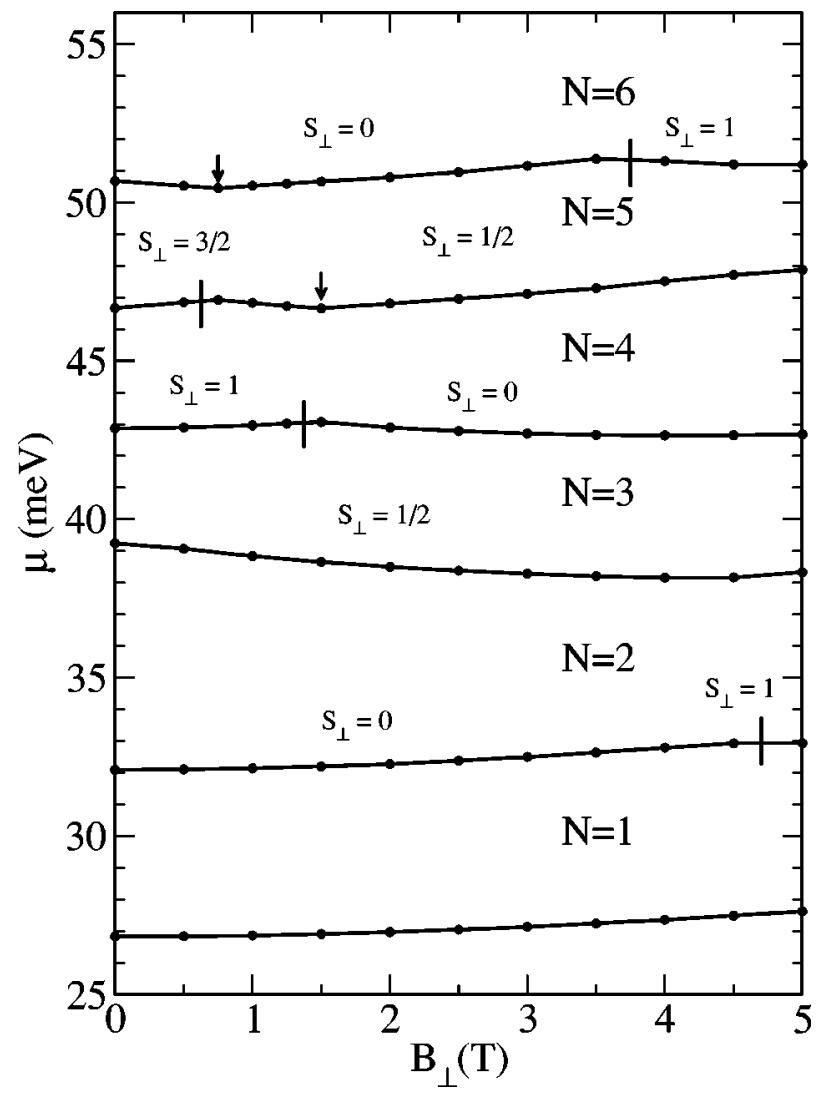

FIG. 8. Theoretical ground state electrochemical potentials in the $B_{\perp}$ case (dots) for $N \leqslant 6$. The lines have been drawn as a to guide the eye. The vertical marks along the $\mu(N)$ lines indicate phase boundaries. The value of $S_{\perp}$ in each phase is given. We have indicated by vertical arrows downward kinks arising from s.p. level crossings in the $N-1$ electron ground state that do not produce phase transitions in the $N$ electron ground state.

ingly, in spite of the lack of any spatial symmetry in the system when a perpendicular field is applied, the s.p. levels are still clearly distributed into shells as in the noninteracting case. ${ }^{5,13}$ Notice also the different splitting between $\uparrow$ and $\downarrow$ states. For saturated (zero) spin $(N=6$ case), this is essentially due to the small Zeeman term, whereas for nonsaturated spin ( $N=5$ case) the splitting is mostly due ${ }^{24}$ to the spin-dependent part of the exchange-correlation energy $W^{\mathrm{xc}}$ term in Eq. (3), and this effect is larger the higher the value of the g.s. spin. This explains the sizable splitting between the two lower lying s.p. levels for $N=5$ up to $B_{\perp} \sim 0.5 \mathrm{~T}$ and the splitting of all the s.p. levels for $N=6$ above $B_{\perp} \sim 3.5 \mathrm{~T}$ (see also Fig. 8).

The calculated g.s. electrochemical potentials are shown in Fig. 8 as a function of $B_{\perp}$. Comparing with Fig. 3(b) it can be seen that the agreement with experiment is good for $3 \leqslant N \leqslant 6$. We have indicated the value of the total $S_{\perp}$ for all the relevant g.s. phases. In the $B=0$ to $5 \mathrm{~T}$ range, there are some $B_{\perp}$ induced changes in $S_{\perp}$, and these give rise to upward kinks [also marked in Fig. 3(b) by solid down triangles]. Some downward kinks, identified by vertical arrows in the $N=5$ and 6 g.s. electrochemical potentials do not correspond to changes in the $N$-electron $S_{\perp}$. They are associated 
with s.p. level crossings between s.p. states of different $\left\langle\Pi_{z}\right\rangle$ value of the $N-1$ electron system. This is the case for $N$ $=5$ at $B_{\perp} \sim 1.5 \mathrm{~T}$ and $N=6$ at $B_{\perp} \sim 0.75 \mathrm{~T}$, as can be seen in Fig. 8 [also marked in Fig. 3(b) by solid circles]. Because of the lack of spatial symmetry in the system, we do not, in general, attempt to identify the (dominant) g.s. configurations. The configurations shown in simple box style in Fig. 3 (b) are the dominant g.s. configurations at $0 \mathrm{~T}$ and they are expected to remain so for small values of $B_{\perp}$. The singlettriplet transition for $N=2$ in Fig. 8 appears at $\sim 4.7 \mathrm{~T}$, a value comparable with that found in the $B_{\|}$case. The $B_{\perp}$ induced singlet-triplet transition in the experimental data is discussed elsewhere. ${ }^{13}$ It can also be seen that for $N=4$, Hund-first-rule-like filling occurs for $B_{\perp}<1.5 \mathrm{~T}$, even if the g.s. configuration is not strictly axially symmetric. Nonetheless, for $N=6$ at $B=0$ we have found an axially symmetric configuration corresponding to a $2 \mathrm{D}$ harmonic oscillator shell-like filling.

\section{SUMMARY}

We have thoroughly discussed the ground-state electrochemical potentials of a few-electron semiconductor artificial QM in the intermediate coupling regime. A detailed comparison between experimental data and LSDFT calculations shows overall a good agreement for both parallel and perpendicular magnetic fields. The agreement is even more remarkable since the frequency $\omega$ of the lateral confining potential has not been used here as a fitting parameter, but rather it has been derived from a law strictly valid for larger values of $N$. Had we used an even smaller value of $\omega$, the agreement would have been even better.

Any sensible comparison with the results of other calculations $^{3,27}$ and with the experimental data should con- sider the strong influence of the confinement on the actual g.s. QM phases. Large $\omega$ values (compared to $\Delta_{\text {SAS }}$ ) obviously favor the occupation of antibonding states. Consequently, decreasing $\omega$ might "wash-out" phase transitions involving antibonding states. For example, see the ${ }^{2} \Pi_{u}^{+}$ $\rightarrow^{2} \Delta_{u}^{-}$transition for $N=5$ or the ${ }^{1} \Sigma_{g}^{+} \rightarrow{ }^{3} \Pi_{g}^{-}$transition for $N=6$ in Fig. 4. We have checked that this is indeed the case when the $N$ dependent confining potential $\hbar \omega=5.78 N^{-1 / 4}$ $(\mathrm{meV})$ of Refs. 3,27 is used. The same is true for the ${ }^{2} \Sigma_{u}^{-}$ $\rightarrow{ }^{2} \Pi_{u}^{+}$transition for $N=3$ (these two states are practically degenerate at $B=0)$. Thus, a comparison between theory and experiment may help to find accurate and realistic values for the effective lateral confining potential.

Analysis of the $B_{\perp}$ case has shown that the $N=2$ singlettriplet transition sensibly occurs at a $\operatorname{similar} B$ value to that in the $B_{\|}$case. In spite of the absence of the strong Landau quantization inherent to the $B \|$ situation, the s.p. levels are, to a large extent, still distributed into shells. We have also found that spin effects arising from the spin dependence of the exchange-correlation energy can dominate those caused by the small Zeeman term as is also the case for a single QD.

\section{ACKNOWLEDGMENTS}

This work was performed under Grants No. BFM200201868 from DGI and No. 2001SGR-00064 from Generalitat of Catalunya, and by the Specially Promoted Research, Grant-in-aid for Scientific Research, and by DARPA-QUIST program (DAAD 19-01-1-0659). F.A. was funded by CESCA-CEPBA Large Scale Facilities through the program "Improving the Human Potential," Contract No. HPRI1999-CT-00071. We are grateful for the assistance of T. Honda with processing the samples, and for useful discussions with A. Emperador, E. Lipparini, and Y. Tokura.
*Electronic address: guy.austing @ nrc.ca

†Electronic address: manuel@ecm.ub.es

${ }^{1}$ F. R. Waugh, M.J. Berry, D.J. Mar, and R.M. Westervelt, Phys. Rev. Lett. 75, 705 (1995); T. Schmidt, R.J. Haug, K.V. Klitzing, A. Förster, and H. Lüth, ibid. 78, 1544 (1997); G. Schedelbeck, W. Wegscheider, M. Bichler, and G. Abstreiter, Science 278, 1792 (1997); R. H. Blick, D. Pfannkuche, R.J. Haug, K.v. Klitzing, and K. Eberl, Phys. Rev. Lett. 80, 4032 (1998); A. Lorke and R. J. Luykenl, Physica B 256-258, 424 (1998); M. Brodsky, N.B. Zhitenev, R.C. Ashoori, L.N. Pfeiffer, and K.W. West, Phys. Rev. Lett. 85, 2356 (2000); M. Bayer, P. Hawrylak, K. Hinzer, S. Fafard, M. Korkusinsky, Z.R. Wasilewski, O. Stern, and A. Forchel, Science 291, 451 (2001).

${ }^{2}$ J. J. Palacios and P. Hawrylak, Phys. Rev. B 51, 1769 (1995); J. Hu, E. Dagoto, and A.H. MacDonald, ibid. 54, 8616 (1996); J. H. Oh, K.J. Chang, G. Ihm, and S.J. Lee, ibid. 53, R13 264 (1996); H. Imamura, P.A. Maksym, and H. Aoki, ibid. 53, 12 613 (1996); O. Mayrock, S.A. Mikhailov, T. Darnhofer, and U. Rössler, ibid. 56, 15760 (1997); H. Tamura, Physica B 249-251, 210 (1998); Y. Asano, Phys. Rev. B 58, 1414 (1998); Y. Tokura, D.G. Austing, and S. Tarucha, J. Phys.: Condens. Matter 11, 6023 (1999); G. Burkard, D. Loss, and D.P. DiVincenzo, Phys. Rev. B 59, 2070 (1999); C. Yannouleas and U. Landman, Phys.
Rev. Lett. 82, 5325 (1999); 85, 2220(E) (2000); Eur. Phys. J. D 16, 373 (2001); L. Martín-Moreno, L. Brey, and C. Tejedor, Phys. Rev. B 62, R10633 (2000); A. Wensauer, O. Steffens, M. Suhrle, and U. Rössler, ibid. 62, 2605 (2000).

${ }^{3}$ M. Rontani, F. Rossi, F. Manghi, and E. Molinari, Solid State Commun. 112, 151 (1999); M. Rontani, F. Troiani, U. Hohenester, and E. Molinari, ibid. 119, 309 (2001).

${ }^{4}$ B. Partoens and F. M. Peeters, Phys. Rev. Lett. 84, 4433 (2000).

${ }^{5}$ Y. Tokura, S. Sasaki, D.G. Austing, and S. Tarucha, Physica E (Amsterdam) 6, 676 (2000).

${ }^{6}$ M. Pi, A. Emperador, M. Barranco, and F. Garcias, Phys. Rev. B 63, 115316 (2001).

${ }^{7}$ D.G. Austing, T. Honda, K. Muraki, Y. Tokura, and S. Tarucha, Physica B 249-251, 206 (1998).

${ }^{8}$ S. Tarucha, D. G. Austing, T. Honda, R.J. van der Hage, and L. P. Kouwenhoven, Phys. Rev. Lett. 77, 3613 (1996).

${ }^{9}$ M. Pi, A. Emperador, M. Barranco, F. Garcias, K. Muraki, S. Tarucha, and D. G. Austing, Phys. Rev. Lett. 87, 066801 (2001).

${ }^{10}$ G. Burkard, G. Seelig, and D. Loss, Phys. Rev. B 62, 2581 (2000).

${ }^{11}$ S. Sasaki, D. G. Austing, and S. Tarucha, Physica B 256-258, 157 (1998).

${ }^{12}$ D. Sánchez, L. Brey, and G. Platero, Phys. Rev. B 64, 235304 (2001). 
${ }^{13}$ S. Sasaki, Y. Tokura, D. G. Austing, K. Muraki, and S. Tarucha (unpublished).

${ }^{14}$ J. P. Perdew and A. Zunger, Phys. Rev. B 23, 5048 (1981).

${ }^{15}$ M. Stopa, Phys. Rev. B 54, 13767 (1996); M. Koskinen, M. Manninen, and S. M. Reimann, Phys. Rev. Lett. 79, 1389 (1997); I.-H. Lee, V. Rao, R. M. Martin, and J.P. Leburton, Phys. Rev. B 57, 9035 (1998); R.N. Barnett and U. Landman, ibid. 48, 2081 (1993).

${ }^{16}$ D.G. Austing, T. Honda, and S. Tarucha, Semicond. Sci. Technol. 11, 388 (1996).

${ }^{17}$ S. Amaha, D.G. Austing, Y. Yokura, K. Muraki, K. Ono, and S. Tarucha, Solid State Commun. 119, 183 (2001).

${ }^{18}$ The frequency of the lateral confining harmonic potential is sometimes made $N$ dependent using as a guide the $r$-expansion near the origin of the Coulomb potential created by a $2 \mathrm{D}$ uniform positive charge distribution (jellium model). This yields $\omega \propto N^{-1 / 4}$. Its use can be reasonably justified for many electrons, which is not the situation in this work. The explicit law we used here for the $b=3.2 \mathrm{~nm} \mathrm{QM}$ is $\hbar \omega=6.91 / N^{1 / 4} \mathrm{meV}$. It has essentially been obtained by imposing that the experimental low$B_{\|}$border in the $N-B_{\|}$plane, at sufficiently large $N$, of the maximum density droplet phase of vertical diatomic artificial molecules is well reproduced. For example, in the strong coupling limit ( $b=2.5 \mathrm{~nm}$ ) see Fig. 3(b) of Ref. 17.

${ }^{19}$ For one single QD, it is worth mentioning the rather succesful attempts have recently been made to obtain the confining potential from the electrostatics of "model" devices with the aim of avoiding the more phenomenological point of view adopted here. See, for example, N. A. Bruce and P. A. Maksym, Phys. Rev. B 61, 4718 (2000); and explicitly for our vertical single quantum dot structures S. Bednarek, B. Szafran, and J. Adamowski, ibid. 64, 195303 (2001); P. Matagne and J.P. Leburton, ibid. 65, 235323 (2002). However, there is still arbitrariness and uncertainly even in these full-blown and impressive calculations, because no model yet exists to accurately include deviations from ideality and randomness which occur in all real dot structures [see also P. Matagne, J.P. Leburton, D.G. Austing, and S. Tarucha, ibid. 65, 085325 (2002)].

${ }^{20}$ I.-H. Lee, Y.-H. Kim, and R.M. Martin, Phys. Rev. B 61, 4397 (2000)

${ }^{21}$ W. H. Press, S. A. Teukolsky, W. T. Vetterling, and B. P. Flannery, Numerical Recipies (Cambridge University Press, Cambridge, 1992).
${ }^{22}$ T. Hoshi, M. Arai, and T. Fujiwara, Phys. Rev. B 52, R5459 (1995).

${ }^{23}$ M. Ferconi and G. Vignale, Phys. Rev. B 50, 14722 (1994).

${ }^{24}$ M. Pi, M. Barranco, A. Emperador, and E. Lipparini, Phys. Rev. B 57, 14783 (1998).

${ }^{25}$ O. Steffens, U. Rössler, and M. Suhrke, Europhys. Lett. 42, 529 (1998).

${ }^{26}$ S.M. Reimann, M. Koskinen, and M. Manninen, Phys. Rev. Lett. 83, 3270 (1999).

${ }^{27}$ B. Partoens and F.M. Peeters, Physica B 298, 282 (2001); Europhys. Lett. 56, 86 (2001).

${ }^{28}$ T.H. Oosterkamp, J.W. Jannsen, L.P. Kouwenhoven, D.G. Austing, T. Honda, and S. Tarucha, Phys. Rev. Lett. 82, 2931 (1999).

${ }^{29}$ L.P. Kouwenhoven, T.H. Oosterkamp, M.W.S. Danoesastro, M. Eto, D.G. Austing, T. Honda, and S. Tarucha, Science 278, 1788 (1997).

${ }^{30}$ The simple schematic box notation in Fig. 3 that represents the molecular states is more intuitive than the full spectroscopic notation used in Fig. 4, although we do not use it in Fig. 4 because of lack of space. Nonetheless, it is easy to go from one notation to the other since each box represents a s.p. level characterized by a radial quantum number $n$ and an angular momentum $l$. The black (gray) arrows denote the thrid component of spin for electrons in bonding (antibonding) states. At $0 \mathrm{~T}$, for both bonding and antibonding states separately, the lowest box is the $(n, l)=(0,0) 1 s$ state, and above that are two boxes, degenerate in energy, which are the $(0, \pm 1) 2 p$ states [see, for example, $N=5$ in Fig. 3(a))]. For the spectroscopic notation, the superscript - of states shown in Fig. 4 is associated with an electron in an antibonding box in Fig. 3(a), and the total $S_{z}$ and $L_{z}$ are readily obtained by adding the spin and orbital angular momentum of all the electrons. Parity ( $g$ or $u$ ) is obtained by multiplying the parities of the s.p. states.

${ }^{31}$ J. P. Dahl, Introduction to the Quantum World of Atoms and Molecules (World Scientific, Singapore, 2001).

${ }^{32}$ The authors of Ref. 27 have identified the vorticity $\mathcal{V}$ with the external magnetic field $\vec{B}$. While this is indeed the case for a homogeneous 2D electron gas, and a fairly good approximation for large QD's, this may not be a good approximation for small systems, as in the present case. CSDFT implementations better suited for small systems are presented in Refs. 26,23-25, for example.

${ }^{33}$ Due to the convention adopted for the s.p. wave functions (Ref. 6) the s.p. orbital angular momentum is actually $-l$. 